\title{
Investigation magnetorheological elastomer elastic deformations under the influence of vibration and electromagnetic load
}

\author{
Andrey Minaev ${ }^{1, *}$, Juri Korovkin ${ }^{1}$, Hammat Valiev $^{2}$, Gennady Stepanov ${ }^{3}$ and Dmitry \\ Borin $^{4}$ \\ ${ }^{1}$ Federal budget - funded research Institute of Machines Science named after A.A. Blagonravov of the \\ Russian Academy of Sciences, Moscow 101990, Russia \\ ${ }^{2}$ Federal budget - funded research Institute of applied mechanics of the Russian Academy of \\ Sciences, Moscow 125040, Russia \\ ${ }^{3}$ State Research Institute for Chemical Technologies of Organoelement Compounds, 111123, Russia \\ ${ }^{4}$ Technische Universität Dresden, Chair of Magnetofluiddynamics, Measuring and Automation \\ Technology, 01062 Dresden, Germany
}

\begin{abstract}
Experimental studies of the magnetorheological elastomer dynamic properties under the influence of vibrations and magnetic fields have been carried out. Amplitude-frequency characteristics of these composites were studied. The computational modeling was made and a device for creation and control of magnetoactive composite movements was developed. The interrelation of the obtained characteristics with the materials microstructure features was deduced.
\end{abstract}

\section{Introduction}

The development of micro - and nanotechnology in many high-tech production fields needs the devices creation for movement and active vibration isolation with extremely stringent requirements for driving dynamic parameters [1]. At present intensive research in various directions for the creation of controlled elastic polymer materials is being conducted. Great attention is directed to the elastomer composites with ferromagnetic fillers. Such composites are able to show intellectual smart properties in response to small applied external magnetic fields. This opens wide opportunities for these materials effective use in human health monitoring, biomedicine, adaptive optics, defense, aerospace, robotics, sports products, etc. In particular there is great interest in the possibile application such composites for artificial muscles creation that mimic the human muscles work. These actuators advantage is contactless, remote action, great speed and high deformation parameters. Some new research results on such materials properties are reported in [2-34]. In order to create and produce this type of materials controlled by a given program, we are

\footnotetext{
* Corresponding author: minaev0804@ya.ru
} 
conducting studies of dynamic properties [35-37] and are developing and justifying technologies for advanced applications magnetorheological materials.

\section{Experimental Methods}

The magnetoactive composites preparation method was described in our works [38 - 40]. The carbonyl iron powders type P 10 modified by hydrophobisator GKZh-94 were used as magnetic filler of CIEL brand silicone polymer matrix. The magnetic filler concentration was $80 \%$ mass. The obtained samples have Young elasticity modulus about $50 \mathrm{kPa}$. The different dynamic test series on a cylindrical sample $3 \mathrm{~cm}$ long and $2.8 \mathrm{~cm}$ in diameter are carried out. The vibration characteristics was carried out at the IMASH vibration testing complex, equipped with instruments that include a setting generator, amplifying equipment and vibration measuring instruments. The vibration amplitude modules and frequency control on the vibrator table at the test sample input were set using virtual programs installed on the computer. The composites surface structure was studied using an atomic force microscope (AFM) EasyScan (Nanosurf, Switzerland), working in a semi-contact mode in air at room temperature. The AFM phase contrast mode was used for the filler particles visualization in the rubber matrix.

\section{Experimental results and discussion}

The vibration test complex contains a dynamic bench, the tested materials - magnetoactive elastomers - were fixed on the table shown in Fig. 1. The softest synthesized composite with high elasticity was tested in this series. The specimen amplitude-frequency characteristics (AFC) were obtained. The performed dynamic tests on vibrostends using the influence of magnetic fields on the magnetorheological elastomers (MAE) samples have shown wide opportunities for changing their resonance characteristics.

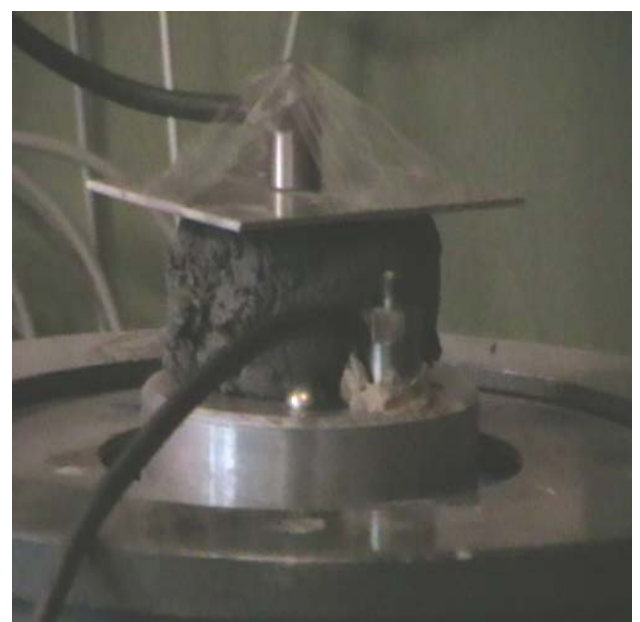

Fig. 1.The magnetoactive elastomer sample mounted on the vibrator table with equipment.

This type specimen testing included determination of the possible resonance frequencies shift under the magnetic field action. Fig. 2 a, b shows the obtained experimental AFC. Here, on the horizontal axis, the sample frequency in hertz $(\mathrm{Hz})$ is shown; on the vertical axis - the voltage in millivolts $(\mathrm{mV})$. Voltages were recorded by piezoaccelerometers mounted on the vibrator table: the input (lower end of the sample) and output (upper end of 
the sample). The voltage $1 \mathrm{mV}$ correspond to accelerations $7,4 \mathrm{~m} / \mathrm{s}^{2}$ The sample maximum attraction force (tear-off) was measured in newtons (H). Fig. 2 a shows the AFC obtained without the magnetic field influence on the test sample; red here shows the sample input AFC; black - shows the output AFC. In this case, the resonance frequencies are close to 35 Hz. Fig. 2 b shows the amplitude-frequency characteristics obtained with relatively weak magnetic field influence on the test sample, the sample force attraction (detachment) was $1.79 \mathrm{~N}$. The black color represents the AFC at the sample input, and the red color represents the sample output. In this experiment the resonance frequency was registered near $70 \mathrm{~Hz}$. Thus, it is shown that exposure of relatively weak magnetic field on the sample leads to a significant resonance frequencies shift: almost twice. Fig. 3 shows the AFC when exposed to the sample by an external load. Input signals on the amplitude-frequency response are black here, output signals are red. The relatively small weight of 20 grams mounted on the sample did not significantly reduce the resonance frequency to $30 \mathrm{~Hz}$.

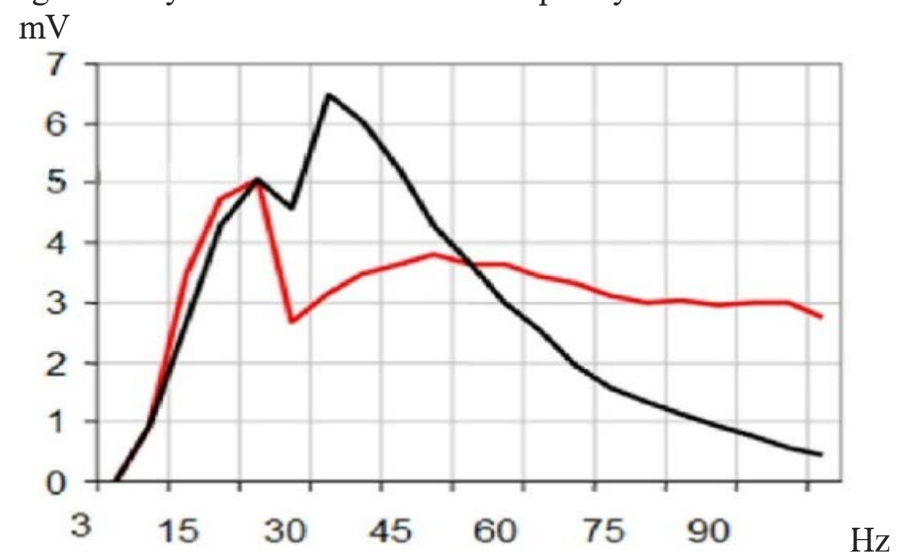

a) Amplitude - frequency characteristics without magnetic field effect on the sample.

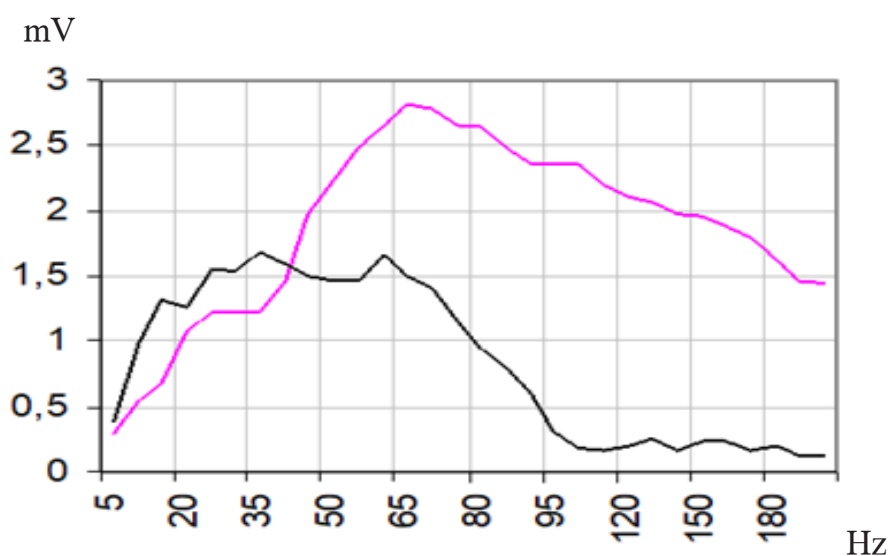

b) Amplitude-frequency characteristics under the influence of a magnetic field on the sample.

Fig. 2. Amplitude - frequency characteristics of the tested sample. 


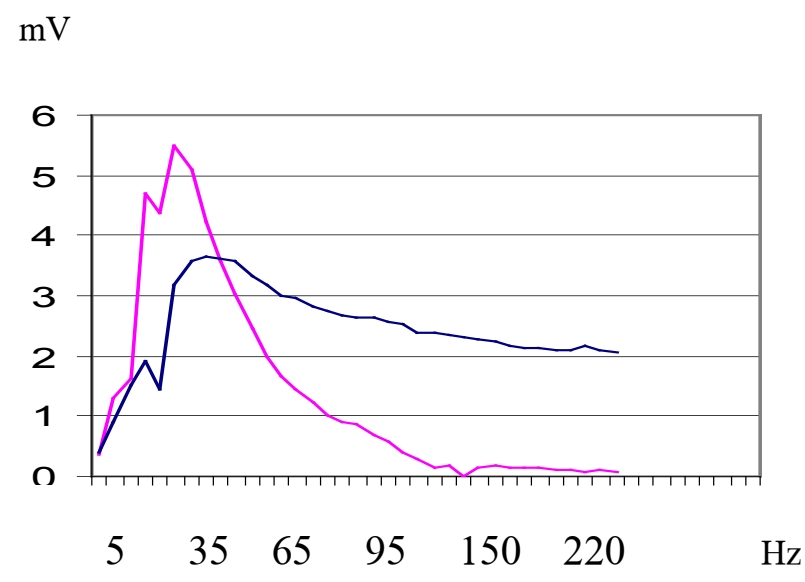

Fig. 3. Amplitude - frequency characteristics, when the weight is mounted on a sample.

Thus, from the conducted tests results it follows that under the insignificant magnetic field influence on the sample the resonance frequency location zone is significantly changed.

The use so controlled magneto-active composites is possible in the hybrid soft robots construction for the capture and non-destructive manipulation of fragile objects, as well as for their maintenance safety by humans. Soft elements motor activation involves moving them in space, which is associated with elastic compression and stretching deformations of their molecular matrix structure similar to soft elements motor activation in living organisms muscles. For this purpose, it is possible to build into the magnetically controlled composites matrix micro solenoids together with micro-dimensional neodymium magnets located at an extremely small distance and coaxially, so that the magnetic induction vectors $\mathbf{B}$ sol and $\mathbf{B}$ mag are on the same axis. The magnitude of the magnetic interaction between solenoid and permanent magnet can be controlled by changing the solenoid magnetic induction from zero to the maximum value. One way to apply the magnetic interaction of solenoid and permanent magnet is to use them as the robot elastic element in the gap between the coaxial solenoid and magnet, deforming it by compression or stretching. magnetic force.

Solenoid and magnet interaction force formula has a form:

$$
F_{\text {sol-mag }}=\frac{B_{\text {sol }} \cdot B_{\text {mag }} \cdot S}{d^{2}}
$$

where: $B_{\text {sol }}$ - magnetic induction of solenoid, $B_{\text {mag }}$ - magnet induction, $S-$ interaction area in $\mathrm{m}^{2}, d-$ distance in $\mathrm{m}$ between solenoid and magnet.

In the solenoid-magnet interaction force formula, the control value is:

$$
B_{\text {sol }}=\mu \cdot \mu_{0} \cdot H_{\text {sol }}=\mu \cdot \mu_{0} \cdot w_{\text {sol }} I_{\text {sol }},
$$

$\mu-$ magnetic air permeability (vacuum), $\mu_{0}=4 \cdot \pi \cdot 10^{-7} \frac{H}{m}$ - magnetic field strength on the solenoid axis, $w_{\text {sol }}$ - number of solenoid turns, $I_{\text {sol }}$ - current in amperes flowing through the solenoid $w_{\text {sol }}$ winding. 
Solenoid current $I_{\text {sol }}$ can be changed from zero to the maximum possible calculated value, changing also the current flow direction, which will change the solenoid magnetic field induction vector direction, i.e. the power lines direction. As a result the direction of the interaction force $F_{\text {sol-mag }}$ between the solenoid and the magnet will also change, which will cause the robot soft element elastic deformation in compression or stretching. It is possible to calculate the interaction force $F_{\text {sol-mag }}$ between a solenoid and a neodymium magnet by having the magnetic induction value of a neodymium magnet where the maximum residual magnetic induction can, for example, be 1.21Tesla.

The proposed activation (deformation) method of the elastomers molecular matrix structure can be made by embedding into their matrix cells ferromagnetic nanoparticles, there movement inside the cells under the magnetic fields influence deforms the entire matrix, and therefore the soft elements structure. In this case the movements of these elements can be software fixed in size and direction and can be programmed to change. In accordance with this the magnetic fields causing the motor deformation of the robot structure parts magnetic active soft elements must be programmable both in size and direction. Such magnetic fields can be created by solenoids or electromagnets and their magnitude depends on the windings number and the current amount flowing through these windings.

The MAE surface structure restructuring under the external permanent neodymium magnet magnetic field action was investigated by atomic force microscopy (Fig. 4 a, b).

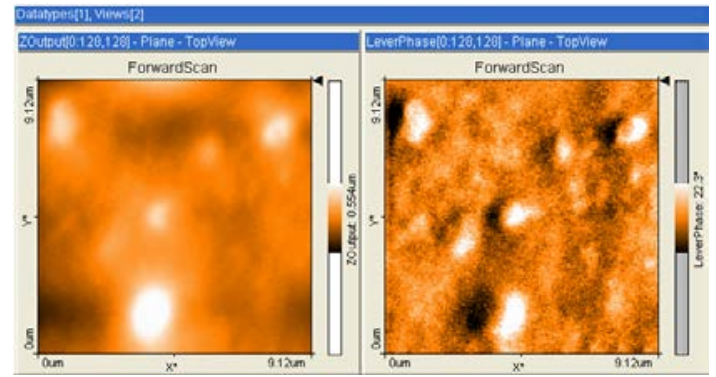

a)

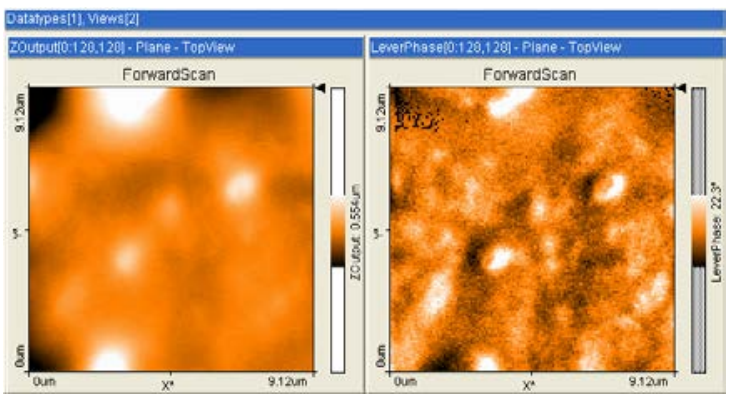

b)

Fig. 4. AFM images of a magnetoactive elastomer surface structure (topography - on the left, phase contrast - on the right): a) without magnetic field;. b) in a magnetic field. Scans: $9.12 \times 9.12$ microns.

Practically spherical morphology, from 1 to 5 microns dimensions of carbonyl iron agglomerates immobilized in elastomer matrix and interatomic distances in a filler grid on the same order were determined. Magnetostrictive displacement of the composite matrix 
together with the filler particles reaches about 2 micrometers under the magnetic field action about 50 millitesla.

Mossbauer effect studies on the isotope nuclei ${ }^{57}$ Fe [40] confirmed the filler microparticles multidomain structure. These data help to understand the mechanism magnetoinduced reinforcement such composites physical and mechanical characteristics. Iron filler microparticles magnetostrictive position redistributions in the rubber matrix definitely cause internal stresses in the composite and, as a consequence, material macroscopic elastic properties strengthening. This is directly visualized in the Fig. $2 \mathrm{a}, \mathrm{b}$ AFC diagrams.

The work was carried out on the subject of state assignment in IPRIM RAS for the period 2019-2021. State registration number: AAAA-A19-119012290177-0.

The work was also supported by RFBR Grant № 19-53-12039.

\section{References}

1. V.A. Glazunov, Modern problems of machine science, Moscow-Izhevsk: Institute for Computer Research, (2015)

2. S.A. Kostrov, V.V.Gorodov, A. M. Muzafarov, E.Yu.Kramarenko,Vysokomol. S o e din, A $62,285(2020)$

3. G. Glavan, W. Kettl, A. Brunhuber, M. Shamonin, I. Drevenšek Olenik, Polymers, 11, 594 (2019)

4. D. Yu. Borin, G. V. Stepanov, E. Dohmen, Arch. Appl. Mechanics, 89, 105 (2019)

5. T. A. Nadzharyan, O. V. Stolbov, Yu. L. Raikher, E. Yu. Kramarenko, Soft Matter, 15, 9507 (2019)

6. T. L. Becker, K. Zimmermann, D. Y. Borin, G. V. Stepanov, P. A. Storozhenko, J. of magnetism and magnetic materials, . 449, 77 (2018)

7. S. Odenbach, Arch. Appl. Mech., 86, 269 (2016)

8. M. Schümann, S. Odenbach, J. of Magn. and Magnetic Materials, 441, 88 (2017)

9. V.V. Sorokin, I. A. Belyaeva, M. Shamonin, E.Yu. Kramarenko, Phys.Rev., E 95, 062501 (2017)

10. Yu. A. Alekhina, L. A. Makarova, T. S. Rusakova, A. S. Semisalova, N. S. Perov, Journal of the Siberian Federal University Ser. Math. Phys., 10, 45 (2017)

11. A. M. Menzel, Archive of Applied Mechanics, 8917 (2019)

12. D. Isaev, A. Semisalova, Y. Alekhina, L.Makarova, N. Perov, Int. J. Mol. Sci., 20, 1457 (2019)

13. D. Romeis, V. Toshchevikov, M. Saphiannikova, Soft Matter, 12, 9364 (2016)

14. M. Cvek, R. Moucka, M. Sedlacik, V. Babayan, V. Pavlínek, Smart Mater. Struct., 26, 095005 (2017)

15. P.S.Tsai, S. Nayak, S. Ghosh, I.K. Puri, AIP Adv., 7, 015003 (2017)

16. R. Moucka, M. Sedlacik, M. Cvek, Appl. Phys. Lett., 112, 122901 (2018)

17. A.V. Bodnaruk, A. Brunhuber, V. M. Kalita, M. M. Kulyk, P. Kurzweil, A. A. Snarskii, M. Shamonin, Polymer, 162, 63 ((2019)

18. Ubaidillah, J. Sutrisno, A. Purwanto, S. A. Mazlan, Adv. Eng. Mater., 17, 563 (2015)

19. M.A. Cantera, M. Behrooz, R.F. Gibson, F. Gordaninejad, Smart Mater. Struct., 26, 023001 (2017)

20. L.A. Makarova, V.V. Rodionova, Y.A. Alekhina, T.S. Rusakova, A.S. Omelyanchik, N.S. Perov, IEEE Trans. Magn., 53, 1 (2017)

21. R. Weeber, M. Hermes, A.M. Schmidt, C. Holm, J. Phys.: Cond. Mat., 30, 063002 (2018)

22. D.Yu. Borin, S. Odenbach, G. V. Stepanov, J. of Magnetism and Magnetic Materials, 470, 85 (2019) 
23. V.M. Kalita, A.A. Snarskii, M. Shamonin, D. Zorinets, Phys. Rev., E 95, 032503 (2017)

24. P.A. Sánchez, T. Gundermann, A. Dobroserdova, S.S. Kantorovich, S. Odenbach, Soft Matter, 14, 2170 (2018)

25. J. Yao, Y. Sun, Y. Wang, Q. Fu, Z. Xiong, Y. Liu, Compos. Science Technol., 162, $170(2018)$

26. Y. Wang, S. Xuan, L. Ge, Q. Wen, X. Gong, Smart Mater. Struct., 26, 015004 (2016)

27. M. Krautz, D. Werner, M. Schrödner, A. Funk, A. Jantz, J. Popp, J. Eckert, A. Waske, J. Magn. Magn. Mater., 426, 60 (2017)

28. B. Xu, C. Paillard, B. Dkhil, L. Bellaiche, Phys. Rev., B 94, 140101 (2016).

29. T. Tian, M. Nakano, J. Intell. Mater. Syst. Struct., 29, 151 (2018)

30. D. Ivaneyko, V. Toshchevikov, M. Saphiannikova, Polymer, 147, 95 (2018)

31. I. Agirre-Olabide, A. Lion, M.J. Elejabarrieta, Smart. Mater. Struct., 26, 035021 (2017)

32. M. Schrödner, G. Pflug, J. Magn. Magn. Mater., 454, 258 (2018)

33. F.H. Sánchez, P. Mendoza Zélis, M.L. Arciniegas, G.A. Pasquevich, M.F. van Raap, Phys. Rev., B 95, 134421 (2017)

34. V.M. Kalita, D.M. Polishchuk, D.G. Kovalchuk, A.V. Bodnaruk, S.O. Solopan, A.I.

Tovstolytkin, S.M. Ryabchenko, A.G. Belous, Phys. Chem. Chem. Phys., 19, 27015 (2017)

35. Yu.V. Korovkin, A.Ya. Minaev, G.V. Stepanov, Russ. Engin. Research, 34, 299 (2014)

36. A. Ya. Minaev, Ju.V. Korovkin, H.H. Valiev, Mechanical Science and Technology Update, IOP Conf. Series: Journal of Physics: Conf. Series 1260, 112021 (2019)

37. A.Ya Minaev, Yu.V. Korovkin, Assembly in mechanical engineering, instrument making, 1, 10 (2018)

38. Kh. Kh. Valiev, A. Ya. Minaev, G. V. Stepanov, Yu. N. Karnet, O. B. Yumashev, J. of Surface Investigation: X-ray, Synchr. and Neutron Techniques, 13, 825 (2019)

39. H.H. Valiev, A. Ya. Minaev, G.V. Stepanov, Ju. N. Karnet, Mechanical Science and Technology Update, IOP Conf. Series: J. of Physics: Conf. Series 1260, 112034 (2019)

40. H H. Valiev, V. M. Cherepanov, Yu. N. Karnet, A. Ya. Minaev, G. V. Stepanov, MIP: Engineering-IOP Conf. Series: Materials Science and Engineering, 862, 022062 ((2020) 